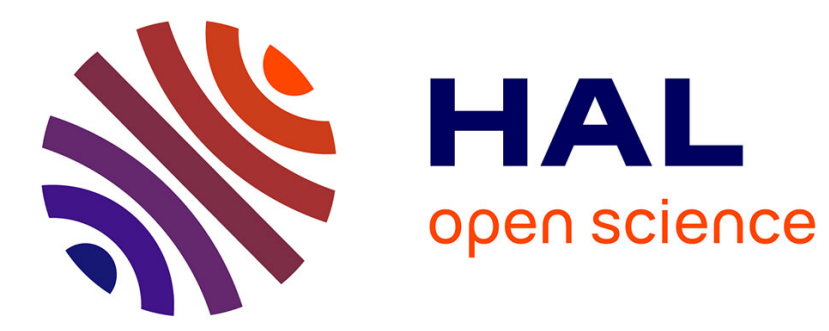

\title{
Stability of quasicrystals: energy, entropy and phason modes.
}

Marc de Boissieu

\section{To cite this version:}

Marc de Boissieu. Stability of quasicrystals: energy, entropy and phason modes.. Philosophical Magazine, 2006, 86 (06-08), pp.1115-1122. 10.1080/14786430500419411 . hal-00513633

\section{HAL Id: hal-00513633 \\ https://hal.science/hal-00513633}

Submitted on 1 Sep 2010

HAL is a multi-disciplinary open access archive for the deposit and dissemination of scientific research documents, whether they are published or not. The documents may come from teaching and research institutions in France or abroad, or from public or private research centers.
L'archive ouverte pluridisciplinaire HAL, est destinée au dépôt et à la diffusion de documents scientifiques de niveau recherche, publiés ou non, émanant des établissements d'enseignement et de recherche français ou étrangers, des laboratoires publics ou privés. 


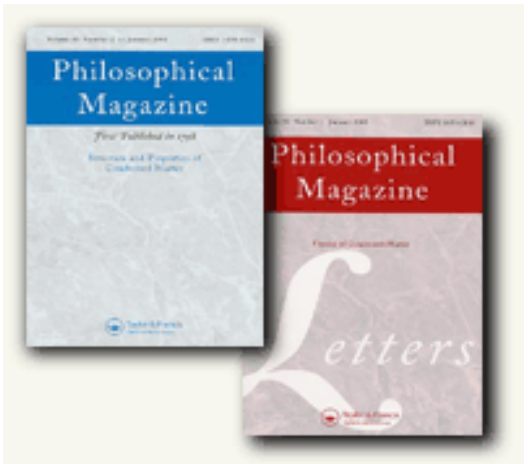

\section{Stability of quasicrystals: energy, entropy and phason modes.}

\begin{tabular}{|r|l|}
\hline Journal: & Philosophical Magazine \& Philosophical Magazine Letters \\
\hline Manuscript ID: & TPHM-05-Jun-0294.R1 \\
\hline Journal Selection: & Philosophical Magazine \\
\hline Date Submitted by the \\
Author: & 02-Sep-2005 \\
\hline Complete List of Authors: & de Boissieu, Marc \\
\hline Keywords: & quasicrystals, phase stability \\
\hline Keywords (user supplied): & Phasons modes \\
\hline &
\end{tabular}

\section{S) ScholaroNE \\ Manuscript Central}


Discussion session ICQ9

Stability of quasicrystals: energy, entropy and phason modes.

\author{
M. de Boissieu \\ Laboratoire de Thermodynamique et Physico Chimie Métallurgique, UMR CNRS 5614, \\ INPG, UJF, ENSEEG, BP 75, 38402 St Martin d'Hères Cedex. \\ The energy and entropy contribution to the quasicrystal stability \\ is an on-going matter of debate. In that respect phason modes \\ play a particular role since these excitations are characteristic of \\ the quasicrystalline long range order. We present some of the \\ results and list open questions to be focused on during the \\ discussion session.
}

Formatted: English (U.K.)

Formatted: English (U.K.)

\title{
1. Introduction
}

The stability of quasicrystals, the way quasiperiodic long range order propagates are still open questions. This is not surprising in view of the complexity of the atomic structure of these phases. It is only recently that a better understanding of the atomic structure by means of structural determination using X-ray or neutron diffraction and High Resolution transmission electron microscopy images has been achieved in a few systems [1-4]. How the structure should be described, whether clusters are entities which have a physical relevance is nevertheless a matter of debate (see W. Steurer, this proceeding).

Concerning the stabilisation mechanism and growth model for quasicrystals, we must admit that we are still relying on very simple models. The two models which are frequently opposed are referred to as the energy and entropy stabilised QC. Their difference relies mainly on the relative weight one is giving to the configurational entropy in the expression of the free energy of the system. This entropy term is related to the phason degree of freedom of the structure. 
The energy stabilised scenario considers that a set of well chosen short range interactions should be enough to give a QC ground state at zero K. This is the case of the 'matching rule' model [5, 6], or of the quasi-unit cell model [7] which in some sense is just a rephrasing of the matching rule model. In the latter one, one considers clusters with some overlapping rules [8].

The entropy stabilised scenario considers that the configurational entropy is a dominant term in the stabilisation mechanism [9]. In this scheme the QC is only stable at elevated temperature and should transform into a crystalline state at lower temperature.

Finally, there are a large number of theoretical and experimental studies on the electronic stabilisation of QC by means of a pseudo-gap at the Fermi level.

In this debate phason modes are playing an important role. We give in the following a brief overview of the theory and experiment on phason modes. A detailed presentation of the hydrodynamics theory of quasicrystals is given in [10] and a review on phason modes in aperiodic crystals can be found in $[11,12]$.

\section{Hydrodynamic theory of $\mathrm{QC}$ and phason modes}

Phason modes are common to all aperiodic crystals. An aperiodic crystal has a definition in term of its Fourier spectrum, which is 'essentially discrete'. It is indexed by a linear combination of $n$ independent linear indices, where $n$ is larger than 3 for 3D crystals. Generally aperiodic crystals are classified in incommensurately modulated crystals (where the modulation can be of the displacive or the chemical type), composites crystals where two subsystems interact, and quasicrystals which have space groups incompatible with lattice translation [13]. As for any classification scheme, there is some arbitrariness, and there are sometime some ambiguities.

The hydrodynamic theory of aperiodic crystals relies on the analysis of the broken symmetry of the system when going from the high to a lower temperature phase as for instance from the liquid to the solid state for QC [10, 14]. Mode counting arguments and symmetry analysis allows then to predict what are the hydrodynamic modes, i.e. modes for which the frequency goes to zero as a power of the wavevector q.

For all aperiodic crystals, the theory predicts that there should be (n-3) phason modes, which in the long-wavelength limit are collective diffusive modes. For icosahedral 
phases this lead to 3 phason modes [10]. Note that the diffusive character of phason modes (i.e. not a propagative mode) is a consequence of the mode counting argument and applies for all aperiodic structures including incommensurately modulated structure $[10,11,15]$.

The two hypotheses necessary for this approach to be valid are best expressed in the high dim picture of quasicrystals:

- The free energy of the system is invariant upon a rigid translation of the cut space.

- The free energy of the system may be expressed as a function of the squared gradient of the 'phonon' and 'phason' strain (or parallel and perpendicular strains).

Whereas the first hypothesis does not lead to much debate, the second one is not obvious a priori and can only be validated on realistic atomistic models and/or by experimental studies.

In this scheme a phason mode can be represented by a sin wave, with a wavevector $\mathbf{q}_{\mathrm{par}}$ in the parallel (physical) space and a polarisation $\mathbf{e}_{\mathrm{per}}$ in the perpendicular space (In the case of icosahedral phases, there are three phason polarisation vectors associated with each $\mathbf{q}_{\text {par }}$ ) (Fig. 1). The 'dispersion' relation of the diffusive phason mode is given by $\quad-i \omega=D_{\text {phason }} q^{2}$, which leads to an exponential time decay of the phason mode as $\exp (-\mathrm{t} / \tau)$, where $\tau^{-1}=\mathrm{D}_{\text {phason }} \mathrm{q}^{2}$ and $\mathrm{D}_{\text {phason }}$ is the phason diffusion constant (see $\mathrm{S}$. Francoual et al., this proceeding ).

\section{[Insert Fig. 1 about there]}

From the hydrodynamic theory, prediction on the shape and intensity distribution of the diffuse scattering due to the equilibrium distribution of phason modes can be made [16-18].

At this point it might be useful to comment on the phason terminology which is used with three quite different meanings in the quasicrystal community: 'phason jump', 'phason strain' and phason modes. I suggest that we should keep the term phason for phason modes only as already proposed by T. Janssen [13]. Indeed it is the only one characteristic of the quasicrystalline (or aperiodic) long range order. It arises from a general definition valid for all aperiodic systems in the hydrodynamic theory: these are long-wavelength collective excitations with a diffusive character. The term 'phason jump' refers to the local rearrangement process at the atomic scale in quasicrystals: it is a single atom process and should be named 'atom flip' or tile flip if one uses a tiling 
description. Note that this process is not characteristic of the quasicrystalline state since it can be found in $1 / 1$ crystalline approximants. The term 'phason strain' refers to a perpendicular shear strain of the periodic space, somehow equivalent to a change of the slope of the cut space. I suggest naming it 'perpendicular' strain (or 'internal' strain if one uses the aperiodic community terminology).

\section{Microscopic models}

The hydrodynamic theory is a continuum theory which does not deal with the microscopic interpretation of phason modes. There are several questions which arise, most of them still open:

- What is the atomic realisation of a phason mode?

- Is the free energy always 'analytic' as a function of phason strain?

- What is the origin of the 'restoring' force (related to the gradient square dependence of the perpendicular strain in the free energy) and of phason modes?

- What is the physics driving the collective diffusive phason modes? Can we evaluate the amplitude of phason modes?

Deleted: $A$

Answering these questions requires simulations or modelling at the atomic level. The main model developed for quasicrystals is the 'random' tiling model [9]. In this case the local process is a 'tile flip' inside a rhombic dodecahedron in the case of the 3D Penrose tiling (see Fig. 2): there are two atomic sites, too close to be occupied simultaneously. In this approach, realistic structures are constructed with clusters on the nodes of the tiling: the 'cluster flip' is obtained by rearranging only a few atoms because in real structure incomplete clusters are interpenetrating.

\section{[Insert Fig. 2 about here]}

Simulations on simple models have shown that $[19,20]$ :

- The free energy is analytic for some $\mathrm{T}$ range (hydrodynamic theory is valid)

- The restoring force is of pure entropy origin: the QC has the largest possibility of configurational re-arrangmeent; any departure from the ideal QC leads to a decrease of the entropy.

In the case of 'matching rules' model, there is a critical $\mathrm{T}$ above which the hydrodynamic theory apply [6]. 
Note that the above models only consider simple Hamiltonian on tilings. (See also Trebin, this proceeding for a 1D model). A more realistic Hamiltonian allowed a simulation of elastic constants as a function of $\mathrm{T}$ [21]. The role of the chemistry (stoechiometry and energy of local environments) and its influence on the free energy when a perpendicular strain is applied has been studied on 2D models ([22, 23]): it was shown that the system still has a phason elasticity described by the hydrodynamic theory, the 'restoring force' being more similar to a chemical potential in this case. The calculation of phason elastic constants on a realistic 3D atomic structure remains however a difficult problem to be solved.

Open question:

- all above plus:

- What is the difference in phason modes for tiling models and cluster models if any?

More elaborated models have been developed in the field of incommensurately displacive modulated structure (DIFFOUR model for instance). In this case there is a rather complete understanding of the physics of phason modes and of the mechanism leading to an incommensurate phase: it is a competition between first and second neighbours interaction leading to a frustration. There is a competition between entropy and energy terms leading to the succession of phases as $\mathrm{T}$ decreases: High symmetry phase, incommensurately displacive modulated phase, 'locked' in phase (equivalent to a rational approximant) [12].

\section{4- Experimental results}

\section{4-1 Atomic flip}

- Quasielestic neutron scattering experiments (Coddens et al.) have evidenced a signal, whose intensity is increasing with the temperature above $600^{\circ} \mathrm{C}$, in icosahedral quasicrystals (AlPdMn and $\mathrm{AlCuFe}$ ). It is interpreted as the result of assisted jump process involving a few ( 2 or 3 ) atoms. The length involved in these jumps is of the order $0.3 \mathrm{~nm}$ [24].

- Abe et al. have observed an anomalous Debye Waller factor by $\mathrm{Z}$ contrast images in the deca phase. The atomic sites where the anomalous Debye Waller is observed
Deleted: - is a tiling the proper description of the structure?I

- Clusters and phason modes (see W. Steurer)?II 
correspond to the border of the atomic surfaces in the 5D model. It thus corresponds to a 'flip site' with a distance of the order 0.1-0.2 nm [3].

\section{4-2 Long-wavelength phason modes:}

Studies on phason modes in quasicrystals have been mainly carried out by diffuse scattering measurements. This experimental approach is completely analogous to the one used for the study of phonons in crystals by Laval, Olmer, Currien and Walker in the 40-50' [25-28]

Most of the results have been obtained in the i-AlPdMn phase for which a complete study has been carried out.

At room temperature there is a large amount of diffuse scattering whose intensity distribution can be interpreted in the hydrodynamic theory framework, using only two phason elastic constants $\mathrm{K} 1$ and $\mathrm{K} 2$ [29, 30]. Their absolute value has also been determined and shown to be independent on the sample annealing treatment [31]. The in situ temperature study shows that the diffuse scattering intensity increases as $\mathrm{T}$ goes from $770^{\circ} \mathrm{C}$ to $500^{\circ} \mathrm{C}$ : this evolution is fully accounted for by a softening of one of the phason elastic constant, a signature of pre-transitional fluctuations [32]. Finally the diffusive collective character of phason modes has been experimentally evidenced in situ using X-ray photon correlation spectroscopy (see Francoual et al., this proceeding) [33]. There is thus a good agreement with the hydrodynamic theory for the i-AIPdMn phase.

Studies for i-AlPdMn with small chemical composition changes [34, 35] (F2 and F2M phase) and on closely related icosahedral phases (i-AlCuFe [36] or i-AlPdRe [37], which are iso structural), all point to the importance of the chemistry and its link with phason fluctuations. It is most likely that the observed phason elasticity is not only connected to a tile configuration, but also to the detailed chemical order/disorder taking place in the quasicrystal. Indeed, if one considers the net effect of a phason mode onto the real structure, this leads to correlated 'atom flip', but also to correlated 'atom specie' exchange (for instance $\mathrm{Al} / \mathrm{Pd}$ or $\mathrm{Al} / \mathrm{Mn}$ ) with respect to the 'ideal' structure. The 'chemical potential' is thus an important ingredient to be taken into account in any realistic simulation of the observed phason elasticity.

The applicability of the hydrodynamic theory on real decagonal phases has been tackled only recently. Indeed, the diffuse scattering intensity distribution (in the quasiperiodic Bragg layer and in between them) is quite complex in most of the 
decagonal phases and has been interpreted as the result of short range order (see the review by Steurer and reference therein [4]). In situ study of the diffuse scattering in the d-AlNiCo phase have been interpreted in term of phason modes and perpendicular strain distributions [38, 39]. Recently, the X-ray diffuse scattering intensity, measured in the quasicrystalline plane of the decagonal AlNiCo phase has been interpreted as resulting from phason modes [40].

Finally, Edagawa et al. have observed by HREM images in situ 'column' flips on a length scale of $2 \mathrm{~nm}$ [41]. The time correlation of these images has been analysed [42]. Although the validity of the hydrodynamics has not been checked, the observed process involves a move of a large number of atoms since the sample thickness is large.

Open questions:

- How reconcile the time scale observed for 'atom flip' and the one observed for collective diffusive phason modes? (The jump process observed by Coddens is much faster than the collective diffusive modes. Are the two observations related to the same process?)

- What is the role of the chemistry and/or chemicaldisorder in this mechanism? It is most likely a key point in the understanding of phason modes and for the QC stability.

\begin{tabular}{l|} 
Deleted: $R$ \\
Deleted: /
\end{tabular}

- Experimental results on diffuse scattering and phason elasticity do not seem to be in complete, agreement with an entropy term arising from a tile configuration alone. Is a tiling an appropriate description of the structure?

- Some phason modes are propagative in incommensurate structures, why not in QC?

\section{5- Thermodynamics- T stability.}

There are very few calorimetric measurements of thermodynamic quantities such as heat of formation, heat of mixing... The systems in which coexist an approximant and a QC are particularly favourable in that respect. There is a need of such comparative measurements which can then be plugged into phase diagram calculations.

T-Stability: The question of the stability of QC (is there a quasicrystalline ground state ?) will certainly be an on going question for a long time and might turn to be just a theoretical one. Experimentally, almost all QC of reasonable quality for which in situ T 
- What other experiments should be carried out to probe the stability of QC?

- In situ temperature studies on more $Q C_{2}$ in particular the CdYb icosahedral type would be very useful to address the $Q C$ stability problem.

- A comparison with other fields of research (order/disorder phase transitions in metallic alloys, aperiodic crystals....) would certainly be useful. For instance, some incommensurately modulated phases display a succession of phases as a function of temperature, which can be understood on the basis of physical models [12].

- The local chemical order and disorder, certainly plays a key role in stabilising the $Q C$. These point should be addressed both experimentally and theoretically with $a b-$ initio calculation (but up to now limited to a few hundred of atoms), or using adapted potential (such as EAM potentials) for simulation on large size systems.

In conclusion, we have seen that long wavelength phason modes have been observed experimentally and have a dynamics in agreement with the hydrodynamic theory. All in-situ temperature studies of the diffraction pattern (including diffuse scattering), point to the importance of an entropy term in quasicrystal stabilisation and to a relation with phason modes. However a detailed description of these phenomena at the atomic level is still lacking. The problems of the quasicrystal stability and of the propagation of the quasiperiodic long range order remain fascinating questions to be solved

Deleted: $T$
Deleted: QC?
$\begin{aligned} & \text { Deleted: Role of ab-initio calculations? } \\ & \text { Larger unit cell calculations? EAM } \\ & \text { potentials? II }\end{aligned}$
-
Deleted:
Deleted: $C$
Deleted: For instance a devil's
staircase has been observed for IMS but
not for $Q C$

Deleted: asas

Formatted: Font: Not Italic 
1

2

3

4

5

6

7

8

9

10

11

12

13

14

15

16

17

18

19

20

21

22

23

24

25

26

27

28

29

30

31

32

33

34

35

36

37

38

39

40

41

42

43

44

45

46

47

48

49

50

51

52

53

54

55

56

57

58

59

60

Acknowledgments: I thank R. Currat and S. Francoual, for the many enlighting discussions we had on phason modes. 
Fig. 1: Simple illustration of a phason mode in the case of a 1D quasicrystal. It is a sinusoidal mode, with a wavevector along the parallel space, and a polarisation of the mode along the perpendicular space. It is not a propagative mode, i.e. its time dependence will be of the form $\exp (-\mathrm{t} / \tau)$.

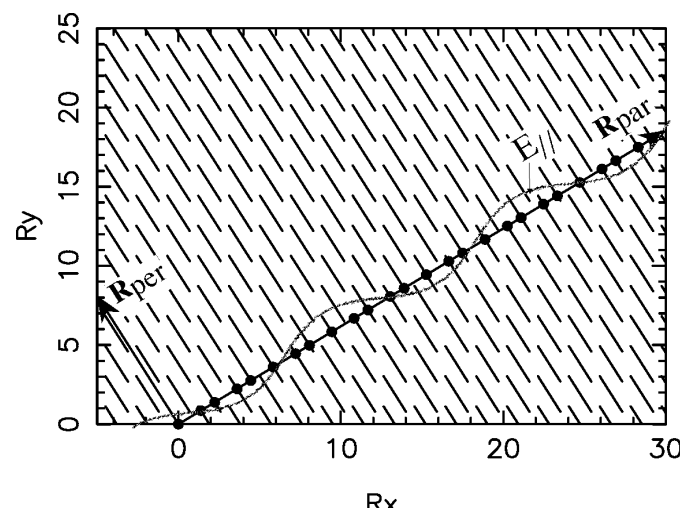

Fig 2: Illustration of the tile flip used in the 3D random Penrose tiling. The two sites inside de rhombic dodecahedron are related by mirror symmetry.
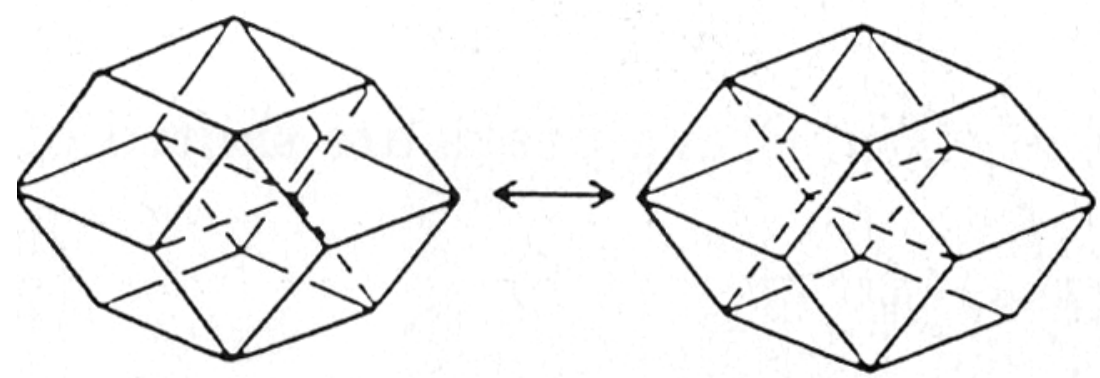


\section{References}

[1] A. Yamamoto, H. Takakura and A.P. Tsai, Phys. Rev. B 6894201 (2003).

[2] D. Gratias, F. Puyraimond, M. Quiquandon and A. Katz, Phys. Rev. B 63 024202/1 (2001).

[3] E. Abe, S.J. Pennycook and A.P. Tsai, Nature 421347 (2003).

[4] W. Steurer, Z. Kristallogr. 219391 (2004).

[5] K. Ingersent, in Quasicrystals : the State of the Art, edited by D. P. Divincenzo and P. J. Steinhardt (World Scientific, 1991), Vol. 11, p. 185.

[6] T. Dotera and P.J. Steinhard, Phys. Rev. Lett. 721670 (1994).

[7] P.J. Steinhardt, H.-C. Jeong, K. Saitoh, M. Tanaka, E. Abe and A.P. Tsai, Nature 36555 (1998).

[8] P. Gummelt, Geometricae Dedicata 621 (1996).

[9] C.L. Henley, in Quasicrystals: the state of the art, edited by D. P. DiVicenzo and P. Steinhardt (World Scientific, 1991), p. 429.

[10] T.C. Lubensky, in Aperiodicity and order, edited by M. V. Jaric (Academic Press, 1988), Vol. 1, p. 199.

[11] M. de Boissieu, R. Currat and S. Francoual, in Physics of Quasicrystals, edited by T. Fujiwara and Y. Ishii (Elsevier Science, (to be published)).

[12] R. Currat and T. Janssen, Sol. Stat. Phys. 41201 (1988).

[13] T. Janssen, O. Radulescu and A.N. Rubtsov, Eur. Phys. J. B 2985 (2002).

[14] P.C. Martin, O. Parodi and P.S. Pershan, Phys. Rev. A 62401 (1972).

[15] Although propagative modes have been observed in a few modulated displacive phases (12), they always are damped modes, with a finite lifetime which remains finite and almost constant as the the wavevector goes to zero. In the long wavelength limit these phason modes are thus overdamped, in agreement with the hydrodynamic theory (see 10 and 11 for a discussion on this point)

[16] M.V. Jaric and D.R. Nelson, Phys. Rev. B 374458 (1988).

[17] Y. Ishii, Phys Rev B-Condensed Matter 455228 (1992).

[18] M. Widom, Phil. Mag. Lett. 64297 (1991).

[19] L.H. Tang, Phys. Rev. Lett. 642390 (1990).

[20] L.J. Shaw, V. Elser and C.L. Henley, Phys. Rev. B 433423 (1991).

[21] M. Mihalkovic and C.L. Henley, Phys. Rev. B 70 09202_1 (2004).

[22] U. Koschella, F. Gahler, J. Roth and H.R. Trebin, J. Alloys Compd. 342287 (2002).

[23] U. Koschella, F. Gahler, J. Roth and H.R. Trebin, J. Non Cryst. Sol. 334-335 276 (2004).

[24] G. Coddens, S. Lyonnard, B. Hennion and Y. Calvayrac, Phys. Rev. B 626268 (2000).

[25] J. Laval, C. R. Acad. Sci., 207169 (1938).

[26] P. Olmer, Acta Cryst. 157 (1948).

[27] H. Curien, Acta. Cryst. 5393 (1952).

[28] C.B. Walker, Phys. Rev. B 547 (1956).

[29] M. de Boissieu, M. Boudard, B. Hennion, R. Bellissent, S. Kycia, A.I. Goldman, C. Janot and M. Audier, Phys. Rev. Lett. 7589 (1995).

[30] M. Capitan, Y. Calveyrac, A. Quivy, J.L. Joulaud, S. Lefebvre and D. Gratias, Phys. Rev. B 606398 (1999). 
[31] A. Létoublon, M. de Boissieu, M. Boudard, L. Mancini, J. Gastaldi, B. Hennion, R. Caudron and R. Bellissent, Phil. Mag. Lett. 81273 (2001).

[32] M. Boudard, M. de Boissieu, A. Létoublon, B. Hennion, R. Bellissent and C. Janot, Europhys. Lett. 33199 (1996).

[33] S. Francoual, F. Livet, M. de Boissieu, F. Yakhou, F. Bley, A. Letoublon, R. Caudron and J. Gastaldi, Phys. Rev. Lett. 91 225501/1 (2003).

[34] M. de Boissieu, M. Boudard, T. Ishimasa, J.P. Lauriat , A. Létoublon, M. Audier, M. Duneau and A. Davroski, Phil. Mag. A 78305 (1998).

[35] A. Létoublon, T. Ishimasa, M. de Boissieu, M. Boudard, B. Hennion and M. Mori, Phil. Mag. Lett. 80205 (2000).

[36] M. Boudard, A. Letoublon, M. de Boissieu, T. Ishimasa, M. Mori, E. Elkaim and J.P. Lauriat, Mat. Sc. Eng. A A294-A296 217 (2000).

[37] M. de Boissieu, H. Takakura, M. Bletry, J.Q. Guo and A.P. Tsai, J. Alloys Compd. 342265 (2002).

[38] H. Abe, H. Saitoh, T. Kusawake, H. Nakao, Y. Matsuo, K. Ohshima, Y. Morikawa and H. Matsumoto, J. Alloys Compd. 342241 (2002).

[39] H. Abe, Y. Ishii and Y. Matsuo, Journal of the Crystallographic Society of Japan 4665 (2004).

[40] M. Kobas, T. Weber and W. Steurer, Submitted (2005).

[41] K. Edagawa, K. Suzuki and S. Takeuchi, Phys. Rev. Lett. 851674 (2000).

[42] K. Edagawa, K. Suzuki and S. Takeuchi, J. Alloys Compd. 342271 (2002). 